\title{
Detection of magnetic circular dichroism on the two-nanometer scale
}

\author{
Peter Schattschneider* and Michael Stöger-Pollach \\ University Service Center for Transmission Electron Microscopy, Vienna University of Technology, Wiedner Hauptstrasse 8-10/052, \\ A-1040 Vienna, Austria \\ Stefano Rubino ${ }^{\dagger}$ \\ Institute for Solid State Physics, Vienna University of Technology, Wiedner Hauptstrasse 8-10/138, A-1040 Vienna, Austria \\ Matthias Sperl, Christian Hurm, and Josef Zweck \\ Institut für Experimentelle und Angewandte Physik, University of Regensburg, D-93047 Regensburg, Germany
}

Ján Rusz

Department of Physics, Uppsala University, P.O. Box 530, S-751 21 Uppsala, Sweden

(Received 17 July 2008; revised manuscript received 18 August 2008; published 18 September 2008)

\begin{abstract}
Magnetic circular dichroism (MCD) is a standard technique for the study of magnetic properties of materials in synchrotron beamlines. We present here a scattering geometry in the transmission electron microscope through which MCD can be observed with unprecedented spatial resolution. A convergent electron beam is used to scan a cross sectional preparation of a Fe/Au multilayer sample. Differences in the energy-loss spectra induced by the magnetic moments of the Fe atoms can be resolved with a resolution of better than $2 \mathrm{~nm}$. This is a breakthrough achievement when compared both to the previous energy-loss MCD resolution (200 $\mathrm{nm}$ ) or the best x-ray MCD experiments (approximately $20 \mathrm{~nm}$ ).
\end{abstract}

DOI: 10.1103/PhysRevB.78.104413 PACS number(s): 75.30.Gw, 78.20.Ls, 68.37.Lp, 79.20.Uv

\section{INTRODUCTION}

Detection of magnetic circular dichroism (MCD) in the electron microscope was first reported in $2006,{ }^{1}$ providing an alternative to X-ray magnetic circular dichroism (XMCD), the standard technique for investigation of spin and orbital magnetic moments in the synchrotron. The fact that spinrelated properties can now be studied with a commercial transmission electron microscope equipped with an energy spectrometer or an energy filter explains the attraction of this technique, named energy-loss magnetic chiral dichroism (EMCD). Contrary to XMCD that measures polarization dependent $\mathrm{X}$-ray absorption cross sections, ${ }^{2-5}$ EMCD exploits faint differences in the double-differential scattering cross section (DDSCS) of fast electrons in the diffraction plane of an electron microscope.

When the method was introduced two years ago, ${ }^{1}$ the spatial resolution was intrinsically limited to $200 \mathrm{~nm}$ by the size of the selected area aperture. To improve that limit, one would have to improve the machinability of transmission electron microscopy (TEM) apertures or develop new scattering geometries. Since then, several laboratories have adopted EMCD,${ }^{6-8}$ and it has become clear that at least two more experimental geometries can be used. The spatial resolution was improved to $30 \mathrm{~nm}$ with the large angle convergent diffraction (LACDIF) method; ${ }^{9}$ here we report a substantial improvement that allows a spatial resolution of $2 \mathrm{~nm}$ to be attained by using convergent electron-beam diffraction in the scanning mode of the TEM. In these geometries, the limiting factors are the aberrations of the microscope and the low count rate resulting from EMCD being essentially an interference effect.

The standard technique for the study of magnetic dichroism on a submicrometer scale is XMCD microscopy (typi- cally with resolutions of about $25-50 \mathrm{~nm}$ ) based on obtaining a circular dichroic signal in combination with imaging optics in a synchrotron. This can be achieved either with electronoptical lenses to form images with photoemitted electrons (XMCD photoemission electron microscopy) or with diffractive $\mathrm{X}$-ray optics ${ }^{4,10-14}$ where a resolution of $15 \mathrm{~nm}$ has been reported. ${ }^{15}$ Also lensless imaging techniques appear to be very promising, in particular, for time-resolved experiments. ${ }^{16}$ For the study of dynamical magnetic properties, EMCD is not suited. Developments of pulsed electron emitters in the TEM may change this situation in the long run.

These techniques have led to considerable progress in the understanding of magnetism in the solid state, and they become increasingly important for the rapidly expanding field of spintronics. The demand for extremely high spatial resolution that arises in this context is met by the intrinsic subnanometer resolution of the TEM.

Here we present a geometry that allows the detection of EMCD in the TEM on the nanometer scale. The method is applied to an epitaxial multilayer of $\mathrm{Fe} / \mathrm{Au}$, demonstrating a spatially resolved MCD signal on a $3 \mathrm{~nm}$ wide Fe layer. An analysis of the signal-to-noise ratio (SNR) shows that the spatial resolution for the detection of MCD is $2 \mathrm{~nm}$ with the chosen setup. This value is likely to be better in a $C_{S}$ corrected microscope.

\section{PRINCIPLES OF THE METHOD}

The DDSCS in the dipole approximation for a geometry with two coherent incident plane waves $\vec{k}_{0}, \vec{k}_{0}^{\prime}$ phase shifted by $\pm \pi / 2[$ Fig. $1(\mathrm{~A})]$ is $^{17,18}$ 


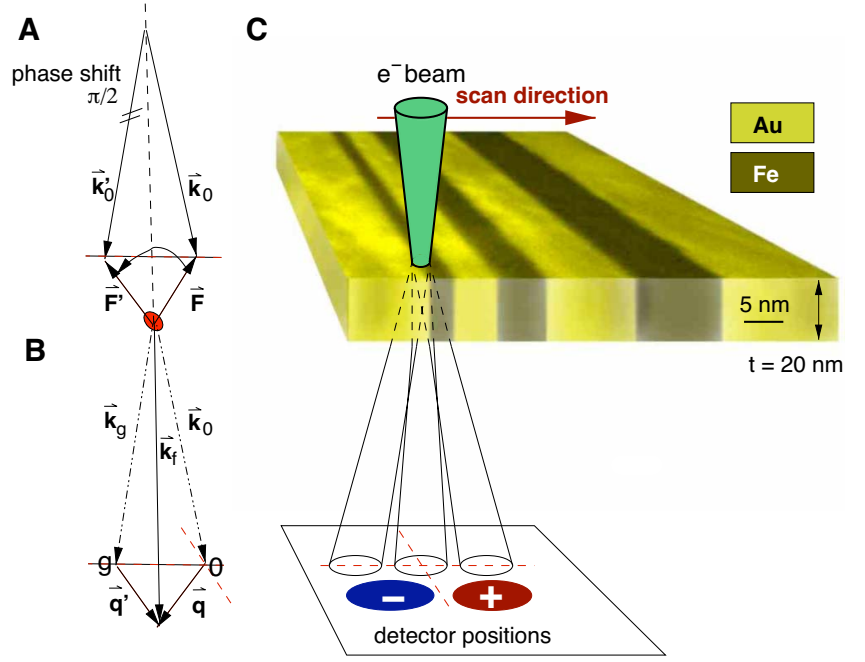

FIG. 1. (Color online) Principle of EMCD. (A) Two coherent incident plane waves, dephased by $\pi / 2$, produce a rotating electric perturbation $\vec{F}$ during the atomic excitation. (B) When crystal diffraction is used, the detector position with respect to the 0 and $g$ beam determines the final scattering direction $\vec{k}_{f}$ and thus $\vec{q}$ and $\vec{q}^{\prime}$. (C) A convergent electron beam is scanned across the $\mathrm{Au} / \mathrm{Fe}$ multilayer sample. The detector is alternatively placed at positions + and - in the diffraction plane selecting two scattering vectors $q \perp q^{\prime}$. The specimen image is a high-angle annular dark field (HAADF) map of the multilayer taken with the $1.7 \mathrm{~nm}$ electron probe.

$\frac{\partial^{2} \sigma}{\partial E \partial \Omega}=\frac{4 \gamma^{2}}{a_{0}^{2}} \frac{k_{f}}{k_{0}}\left(\frac{S(\vec{q}, \vec{q}, E)}{q^{4}}+\frac{S\left(\vec{q}^{\prime}, \vec{q}^{\prime}, E\right)}{q^{\prime 4}} \mp 2 \frac{\Im\left[S\left(\vec{q}, \overrightarrow{q^{\prime}}, E\right)\right]}{q^{2} q^{\prime 2}}\right)$,

where

$$
S\left(\vec{q}, \vec{q}^{\prime}, E\right):=\sum_{i, f}\langle f|\vec{q} \cdot \vec{R}| i\rangle\left\langle i\left|\overrightarrow{q^{\prime}} \cdot \vec{R}\right| f\right\rangle \delta\left(E_{i}-E_{f}+E\right)
$$

is the mixed dynamic form factor, ${ }^{19,20} \vec{k}_{f}$ is the final scattering wave vector (defined by the detector position), $\vec{q}=\vec{k}_{f}$ $-\vec{k}_{0}$ and $\vec{q}^{\prime}=\vec{k}_{f}-\vec{k}_{0}^{\prime}$ are the wave vector transfers, $\gamma$ is the relativistic factor, $a_{0}$ is the Bohr radius, and $E$ is the energy lost by the fast electron. Using the crystal lattice as a beam splitter the coherent incident waves $\vec{k}_{0}, \vec{k}_{0}^{\prime}$ are replaced by $\vec{k}_{0}, \vec{k}_{g}=\vec{k}_{0}+\vec{g}$ with a reciprocal-lattice vector $\vec{g}$, as drawn in Fig. 1(B).

For an atom at the origin the quantity $S$ possesses an imaginary part if the atom has a net magnetic moment $\vec{M}$, which in the present case is forced to be out of the specimen plane and parallel to the optical axis of the TEM by the strong magnetic field $(\approx 2 \mathrm{~T})$ of the objective lens, ${ }^{21}$

$$
\Im\left[S\left(\vec{q}, \vec{q}^{\prime}, E\right)\right] \propto\left(\vec{q} \times \vec{q}^{\prime}\right) \cdot \vec{M} .
$$

The dichroic signal is the difference of two spectra [Eq. (1)] obtained by reversing the sign of the third (interference) term. In the difference spectrum the first two terms cancel; only the third one remains. The triple product is nonzero because the wave vector transfers $\vec{q}$ and $\vec{q}^{\prime}$ are nearly perpendicular to the optical axis, as their $z$ component (due to the energy lost in the ionization) is considerably smaller than the $x, y$ components set by positioning the detector in the diffraction plane.

The helicity of the excitation is changed by placing the detector in the two positions in the diffraction plane [Fig. $1(\mathrm{C})]$ thus selecting two scattering vectors $q \perp q^{\prime}$ of equal length. Shifting the detector from the "+" to the "-" position in Fig. 1(B) changes the sign (but not the magnitude) of the vector product $\vec{q} \times \vec{q}^{\prime}$ and thus of the interference term in Eq. (1).

The equivalence with XMCD can be understood when considering that the inelastic interaction with a given target atom is Coulombic. The perturbation, leading to an electronic transition for an atom at the origin, is an electric field $\mathbf{F} \propto \mathbf{q} e^{i(\omega t+\phi)}$ (and similarly for $\mathbf{q}^{\prime}$ ), with $\hbar \omega=E$, the energy lost by the probe electron in the transition. By forcing the two coherent plane waves (0 and $g$ in Fig. 1) to exhibit a phase difference $\delta \phi=\phi-\phi^{\prime}=\pi / 2$, the electric perturbation vector $\vec{F}+\vec{F}^{\prime}$ at the atomic site rotates clockwise in a plane with surface normal $\vec{q} \times \vec{q}^{\prime}$, thereby forcing a chiral transition obeying the selection rule $\Delta m=+1$ (equivalent to the absorption of a photon with positive helicity). When shifting the detector from position + to - in Fig. 1(C), the vector $\vec{q}$ $\times \vec{q}^{\prime}$ changes sign, the perturbation field rotates counterclockwise, and the chirality of the transition is reversed. As with XMCD, the measured difference spectrum is the dichroic signal.

This ideal situation is never met in practice because it assumes a pointlike detector in the diffraction plane measuring a signal from two monochromatic plane waves ionizing an atom at the origin, without any other interaction. Dynamical electron diffraction from the crystal lattice causes a variation of the phase difference $\delta \phi$ along the electron trajectory with a periodicity given by the extinction distance ${ }^{22}$ (which appears as a beating effect in the intensity known as Pendellösung in electron microscopy). Therefore, even using two nearly monochromatic plane waves, the EMCD signal would always be reduced with respect to XMCD. The favorable phase relation can be approximately maintained over a thickness range of half an extinction distance $(20 \mathrm{~nm}$ in $\mathrm{Fe}$ for the 200 reflection; however, differences up to $5 \%$ are expected for samples as thick as $75 \mathrm{~nm}$ ). Specimens should therefore be prepared for electron microscopy with appropriate thickness. Integration over convergence and collection angles in the microscope and/or detector system induces additional variations in the phase shift between the coherent partial waves, and the presence of secondary Bragg spots other than 0 and $g$ has a complex influence on the dichroic component of the signal.

Diffraction on the crystal lattice, at first view detrimental to the dichroic signal, can be turned to advantage when one realizes that the phase shift between the 0 and the $g$ wave can be tuned by varying the excitation error. Moreover, the lattice periodicity automatically serves as a phase-lock amplifier, creating equal-phase shifts in each elementary cell.

When we extend this formalism to the realistic case of many Bragg scattered waves, Eq. (1) is replaced by 

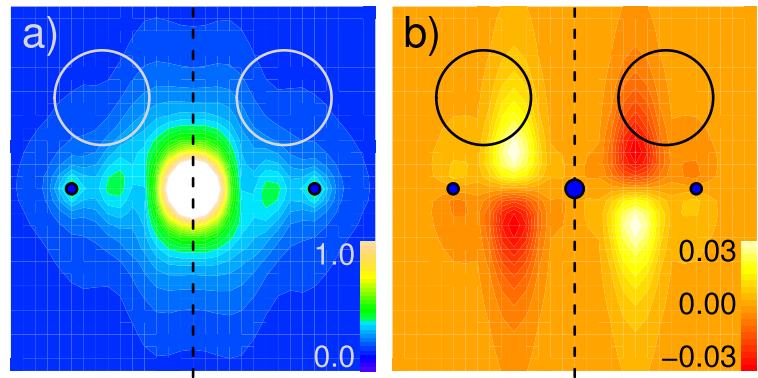

FIG. 2. (Color online) (A) Fe $L_{3}$ signal in the diffraction plane. Symmetric three-beam case $g=(200), t=22 \mathrm{~nm}$, for parallel illumination, which is similar to the LACDIF condition described in the text. The three diffraction spots $-g,(000)$, and $g$ are marked by small circles (blue). The detector positions are marked with large circles. (B) The dichroic signal in the diffraction plane is obtained by subtracting the signal at a point in the left half plane from the signal at the mirror point in the right half plane. Maximum values are $\pm 15 \%$ relative. The dashed vertical line in both panels is a mirror plane for the cubic crystal, yet the symmetry across the plane is broken by the presence of magnetism.

$$
\frac{\partial^{2} \sigma}{\partial E \partial \Omega}=\frac{4 \gamma^{2}}{a_{0}^{2}} \frac{k_{f}}{k_{0}} \sum_{i \leq j} 2 \Re\left[A_{i j} \frac{S\left(\vec{q}_{i}, \vec{q}_{j}, E\right)}{q_{i}^{2} q_{j}^{2}}\right],
$$

where the scattering vectors are enumerated according to the Bragg scattered plane waves in the elastic diffraction pattern. The coefficients $A_{i j}$ are calculated in the framework of dynamical electron-diffraction theory. ${ }^{22}$ Figure 2 shows the simulated Fe $L_{3}$ signal and the relative dichroic signal in the diffraction plane in the symmetric three-beam case $[g$ $=(200), t=22 \mathrm{~nm}]$ for parallel illumination. The detector positions are marked with circles.

\section{EXPERIMENTAL PROCEDURE}

It is found that the dichroic signal is rather robust with respect to variations in detection angle, with only the prefactor varying in magnitude. ${ }^{9}$ Since the incident and the outgoing beams appear symmetrically in the expressions for the prefactors, ${ }^{22}$ the same robustness must hold for the incident electron. We had therefore reason to hope that a converged beam with a convergence angle of the same magnitude as the collector angle would perform almost as well as an incident plane wave. It was therefore tempting to replace the LACDIF
(Ref. 23) by a convergent beam diffraction geometry [Fig. $1(\mathrm{C})]$; here, the specimen remains in eucentric position, and the signal is taken in the diffraction plane. Differently from previously reported geometries ${ }^{1,9}$ the crystal is tilted to a three-beam case (i.e., exciting equally the $+g$ and $-g$ beams). This setup has a symmetry plane perpendicular to $\vec{g}$ passing through the 0 beam (Fig. 2). The diffraction pattern, consisting now of broad Bragg disks instead of sharp pointlike spots, is then electronically shifted such that the detector is placed at symmetric positions, labeled - and + relative to a line perpendicular to the $\mathbf{g}$ vector and passing through (000). As opposed to the two-beam case this geometry has the advantage that one avoids any spectral difference not related to dichroism. The EMCD signal has a spatial resolution given by the beam diameter which cannot be reduced below a certain limit because spectra obtained with smaller electron probes have a lower spectral intensity and low SNR. Experimentally it was found that a nominal spot size of $1.7 \mathrm{~nm}$ yielded a signal strong enough for detection of EMCD in $\mathrm{Fe}$.

In order to reliably determine the spatial resolution, a test specimen was produced by means of molecular-beam epitaxy. First a $0.8 \mathrm{~nm}$ Fe thin film was grown on a (001)-GaAs substrate followed by $25 \mathrm{~nm}$ of $\mathrm{Au}$. Then successive Fe and $\mathrm{Au}$ layers were stacked as the following: $3 \mathrm{~nm} \mathrm{Fe}, 5 \mathrm{~nm} \mathrm{Au}$, $6 \mathrm{~nm} \mathrm{Fe}, 10 \mathrm{~nm} \mathrm{Au}, 10 \mathrm{~nm} \mathrm{Fe}, 21 \mathrm{~nm} \mathrm{Au}$, and $31 \mathrm{~nm} \mathrm{Fe}$ covered with a $25 \mathrm{~nm}$ Au protection layer.

The sample was then prepared in cross section by mechanical grinding and ion polishing. In order to avoid contamination of the sample in the $200 \mathrm{keV}$ electron beam, the sample was plasma cleaned in $5 \mathrm{~N}$ Ar atmosphere directly before inserting it into the microscope. No oxygen was detected during the electron-energy-loss spectroscopy (EELS) characterization.

The specimen was first oriented in zone axis conditions, with the $\mathrm{Au} / \mathrm{Fe}$ interfaces projecting in the TEM image. Then a symmetric three-beam case was set up, tilting the specimen by roughly $5^{\circ}$ off the zone axis such that the interfaces were still projecting, resulting in the excitation of the $( \pm 200)$ spots. The specimen was characterized with $Z$-contrast imaging and high-resolution TEM shown in Figs. 1(C) and 3(A).

The EMCD measurements were performed in the scanning mode of the TEM using the focused probe for two subsequent line scans of the same region, one for each detector position (+ and -). Each line scan, consisting of 20 spectra with a nominal separation of $0.9 \mathrm{~nm}$ for a total of $17 \mathrm{~nm}$ scan length, started in the first $25 \mathrm{~nm}$ Au layer and proceeded on
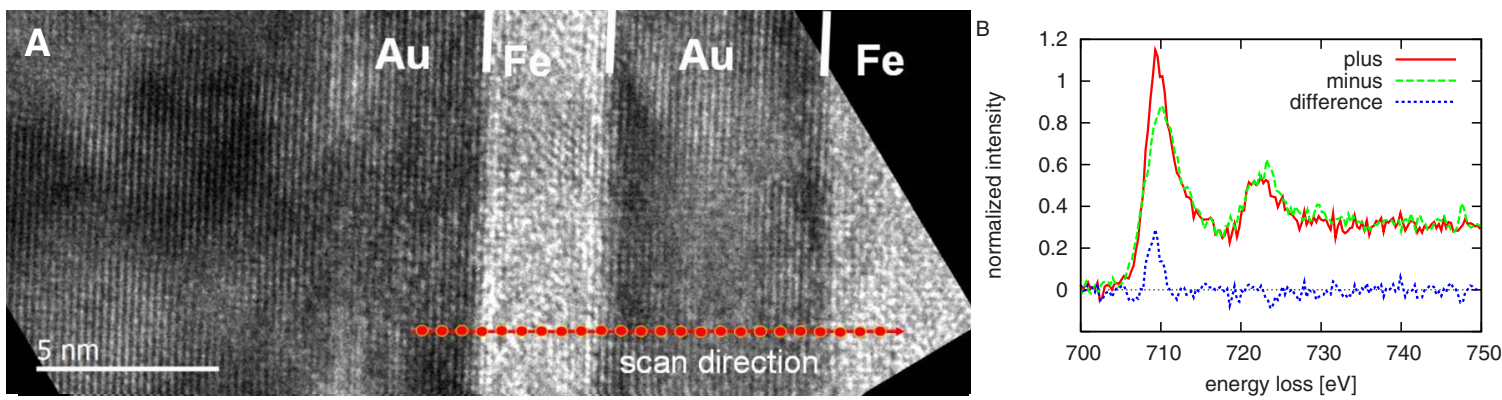

FIG. 3. (Color online) (A) High-resolution TEM image of the investigated area; the shape and position of the beam during the scan are indicated by the superimposed circles. (B) Spectra from the middle of the $3 \mathrm{~nm}$ Fe layer. The difference is the dichroic signal. 
A

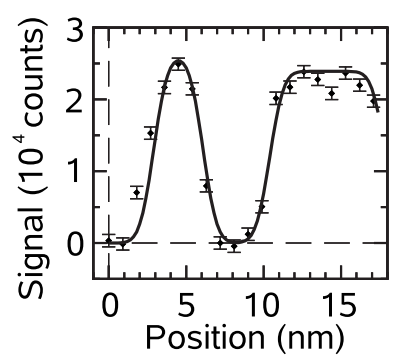

$\mathrm{B}$

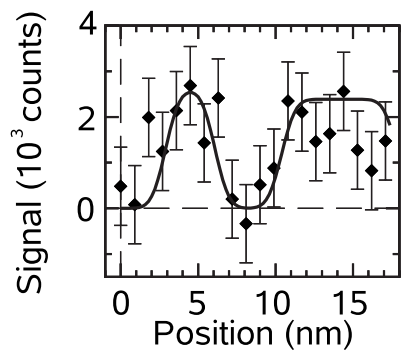

FIG. 4. (A) Profile of the line scanned in Fig. 3. The experimental points are the integrated Fe signal (sum of the + and - spectra) at the $L_{3}$ edge (707.9-713.9 eV). The best fit with a Gaussian spot shape gives a FWHM of $1.66 \mathrm{~nm}$ for the spot. The error bars are $3 \sigma=855$ counts. (B) Corresponding line profile of the dichroic signal (difference of the + and - spectra) integrated at the $L_{3}$ edge.

a straight line perpendicular to the $\mathrm{Au} / \mathrm{Fe}$ interfaces across the first $3 \mathrm{~nm}$ Fe layer, the $5 \mathrm{~nm} \mathrm{Au}$, the $6 \mathrm{~nm} \mathrm{Fe}$, and ended close to the interface with the $10 \mathrm{~nm}$ Au layer [Fig. 3(A)]. At each point an energy-loss spectrum at the $\mathrm{Fe} L_{2,3}$ edge was acquired, with $10 \mathrm{~s}$ acquisition time. These values were chosen to have the highest signal intensity allowed by the specimen and beam drift. The + and - spectra for the point in the middle of the first Fe layer are shown with their difference, representing the dichroic signal [Fig. 3(B)].

\section{RESULTS AND DISCUSSION}

In Fig. 4 the spectral intensity $(A)$ and the dichroic signal (B), integrated over the $L_{3}$ edge from 707.9 to $713.9 \mathrm{eV}$, are shown. The Fe and Au layers are clearly resolved, thus demonstrating a spatial resolution of at least $3 \mathrm{~nm}$.

For the determination of the effective resolution of the EMCD experiment a Gaussian spot profile sweeping across the $\mathrm{Au} / \mathrm{Fe}$ multilayer is assumed. A least-squares fit to the experimental values yields a variance of $\sigma^{2}=1.0 \mathrm{~nm}^{2}$ which translates into a Gaussian full width at half maximum (FWHM) of $1.66 \mathrm{~nm}$. This proves that the factor limiting the resolution is indeed the spot size; delocalization ${ }^{24}$ or nonprojecting interfaces are negligible in the present case. The deviations from the fit function in the leftmost slope in the figure are caused by inconstant drift of the specimen during the scan; variations on the plateau to the right stem from faint thickness variation resulting in changes in the peak height.

We conclude that the Fe signal can be detected with a resolution limit of $\leq 1.7 \mathrm{~nm}$ in the present experiment. Since the EMCD is a difference of Fe signals, its theoretical geometric resolution must be the same. But this is only true for the same SNR. The smaller SNR in the EMCD signal reduces this limit. Nonetheless, the EMCD signal is clearly visible across the $3 \mathrm{~nm}$ Fe layer in Fig. 4(B). The dichroic signal at the $L_{2}$ edge is spread over a larger energy range due to its shorter core-hole lifetime, corresponding to a larger Lorentzian broadening of the $L_{2}$ compared to $L_{3}$ edge. Moreover, the orbital to spin moment ratio is expected to be small but positive for Fe atoms; according to the sum rules ${ }^{21}$ this

means that the integrated area under the $L_{2}$ peak is smaller than the one under the $L_{3}$ peak. The theoretical peak of the dichroic signal at the $L_{2}$ edge is thus expected to be between 800 and 1200 counts, which is statistically insignificant considering the given $3 \sigma$ interval. We note in passing that the statistics of background subtraction can enhance the $L_{3}$ signal at the expenses of the $L_{2}$ signal or vice versa.

The advantage of the geometry presented here lies in the improved use of symmetry and in the optimization of the illumination and acquisition process that enabled the extension to the scanning mode of the TEM. The outcome is atomspecific magnetic characterization with unprecedented spatial resolution. More in detail, the three-beam geometry allows us to acquire two spectra at detector positions connected by a mirror plane, which (unlike the two-beam case used previously) is a true symmetry operation of the whole measurement system. Not only detector positions are symmetric but also the incoming beam lies on the mirror-symmetry plane and the crystal lattice is symmetric with respect to the same plane. This leads to equal dynamical effects at both detector positions (dephasing of wave fronts) and to the same background signals.

It should be noted that cubic crystals such as the one used in the experiment are not expected to show any difference in spectra acquired at those detector positions because of their high symmetry. It is only because of the pseudovectorial nature of the sample's magnetization that a spectral difference arises. In this geometry, spectral differences are caused only by magnetic effects (and noise or inaccuracies ${ }^{25}$ ).

Sum rules ${ }^{21}$ have shown that spectra can be acquired at any position in diffraction plane when the symmetry requirements (see above) are fulfilled. Therefore large collection angles are not an obstacle, as a simple calculation of the diffraction patterns and dichroic maps have shown. It is true that the spread in $k_{f}$ can reduce the EMCD percentage of the spectra, but this reduction in percentage is traded for a significant increase in total signal and results in better EMCD SNR overall.

Similarly, a symmetrical spread of incoming beam wave vectors $\left(k_{0}\right)$ is also allowed. In the past it was shown that the improved SNR could lead to better spatial resolution and this was used here in combination with theoretical results to extend the EMCD technique to scanning transmission electron microscopy (STEM) mode, where the electron beam is focused on a small spot and scanned across the sample. Smaller spot sizes or high beam intensities require more convergent beams. This in turn would reduce the relative variation of the spectral signal due to dichroism. For example, an electron beam with $1 \mathrm{mrad}$ convergent angle could give 100 counts at the $L_{3}$ peak with $10 \%$ dichroism (variation), whereas a 5 mrad convergent beam could give 10000 counts at the $L_{3}$ peak with $5 \%$ dichroism. Assuming Poissonian noise, the EMCD SNR in the first case is 1 and in the second case is 5 . It can be seen that as we need to detect differences in EELS spectra, the demand on the SNR is more stringent. This means that, in some cases, it would be possible to detect an element but not its EMCD signature (or lack thereof).

Apart from these physical developments, the technique itself will have considerable consequences in near futurefor high-resolution atom-specific magnetic studies. We pro- 
vide a method to approach a whole new class of physical problems to be studied. In all previous works published on EMCD, spectra were acquired from a single region in the specimen. The only possibility to study different features in the specimen was to repeat it on another part of the sample. With the STEM mode, line scans are possible, which means that magnetic properties of multilayers can be investigated in a matter of minutes and directly related to high-resolution images. This brings us a step closer to the realization of EMCD imaging capable of revealing magnetic contrast. Moreover, it is an experiment with a cross sectional magnetic multilayer specimen, thus approaching real problems of nanomagnetism.

\section{CONCLUSIONS}

In summary, we have measured an EMCD signal in the STEM mode of the electron microscope. As compared to the original EMCD setup this opens the exciting possibility to map magnetic moments with a lateral resolution of better than $2 \mathrm{~nm}$. This constitutes a breakthrough for the study of nanomagnetism at interfaces and boundaries. The main limiting factors in this experiment are specimen drift and beam instability, which set upper bounds on the collection time of the spectra. If drift can be reduced the dwell time could be increased, thereby lowering the noise level and allowing smaller spots. With the new generation of $C_{S}$ corrected (scanning) TEMs it is likely to achieve subnanometer resolution in EMCD spectrometry.

\section{ACKNOWLEDGMENTS}

The authors acknowledge N. J. Zaluzec, C. Hébert, and J. Verbeeck for programming the script for the electronic shift of the diffraction pattern and E. Carlino, L. Felisari, F. Maccherozzi, and P. Fischer for fruitful discussions. P.S. and S.R. acknowledge funding from the European Union under Contract No. 508971 (FP6-2003-NEST-A) "CHIRALTEM."
*Also at Institute for Solid State Physics, Vienna University of Technology, Wiedner Hauptstrasse 8-10/138, A-1040 Vienna, Austria.

${ }^{\dagger}$ Also at Department of Engineering, Uppsala University, P.O. Box 534, S-751 21, Uppsala, Sweden; stefanorubino@yahoo.it

${ }^{*}$ Also at Institute of Physics, Academy of Sciences of the Czech Republic, Na Slovance 2, CZ-182 21 Prague, Czech Republic.

${ }^{1}$ P. Schattschneider, S. Rubino, C. Hébert, J. Rusz, J. Kuneš, P. Novak, E. Carlino, M. Fabrizioli, G. Panaccione, and G. Rossi, Nature (London) 441, 486 (2006).

${ }^{2}$ G. Schütz, W. Wagner, W. Wilhelm, P. Kienle, R. Zeller, R. Frahm, and G. Materlik, Phys. Rev. Lett. 58, 737 (1987).

${ }^{3}$ C. T. Chen, F. Sette, Y. Ma, and S. Modesti, Phys. Rev. B 42, 7262 (1990)

${ }^{4}$ J. Stöhr, B. D. Hermsmeier, M. G. Samant, G. R. Harp, S. Koranda, D. Dunham, and B. P. Tonner, Science 259, 658 (1993).

${ }^{5}$ P. Fischer, T. Eimüller, G. Schütz, P. Guttmann, G. Schmahl, K. Pruegl, and G. Bayreuther, J. Phys. D 31, 649 (1998).

${ }^{6}$ B. Warot-Fonrose, F. Houdellier, M. J. Hÿtch, L. Calmels, V. Serin, and E. Snoeck, Ultramicroscopy 108, 393 (2008).

${ }^{7}$ L. Calmels, F. Houdellier, B. Warot-Fonrose, C. Gatel, M. J. Hÿtch, V. Serin, E. Snoeck, and P. Schattschneider, Phys. Rev. B 76, 060409(R) (2007).

${ }^{8}$ P. A. van Aken, L. Gu, D. Goll, and G. Schütz, Microsc. Microanal. 13, 426 (2007).

${ }^{9}$ P. Schattschneider, C. Hébert, S. Rubino, M. Stöger-Pollach, J. Rusz, and P. Novák, Ultramicroscopy 108, 433 (2008).

${ }^{10}$ C. M. Schneider, K. Holldack, M. Kinzler, M. Grunze, and H. P. Oepen, Appl. Phys. Lett. 63, 2432 (1993).

${ }^{11}$ J. Stöhr, H. A. Padmore, S. Anders, T. Stammler, and M. R. Scheinfein, Surf. Rev. Lett. 5, 1297 (1998).

${ }^{12}$ B. P. Tonner and G. R. Harp, Rev. Sci. Instrum. 59, 853 (1988).
${ }^{13}$ H. Ohldag, T. J. Regan, J. Stöhr, A. Scholl, F. Nolting, J. Lüning, C. Stamm, S. Anders, and R. L. White, Phys. Rev. Lett. 87, 247201 (2001).

${ }^{14}$ S. Imada, S. Suga, W. Kuch, and J. Kirschner, Surf. Rev. Lett. 9, 877 (2002).

${ }^{15}$ D. H. Kim, P. Fischer, W. Chao, E. Anderson, M.-Y. Im, S.-C. Shin, and S.-B. Choe, J. Appl. Phys. 99, 08H303 (2006).

${ }^{16}$ S. Eisebitt, J. Lüning, W. F. Schlotter, M. Lörgen, O. Hellwig, W. Eberhardt, and J. Stöhr, Nature (London) 432, 885 (2004).

${ }^{17}$ C. Hébert and P. Schattschneider, Ultramicroscopy 96, 463 (2003).

${ }^{18} \mathrm{P}$. Schattschneider and J. Verbeeck, Ultramicroscopy 108, 407 (2008).

${ }^{19}$ H. Kohl and H. Rose, Adv. Electron. Electron Phys. 65, 173 (1985).

${ }^{20}$ P. Schattschneider, M. Nelhiebel, and B. Jouffrey, Phys. Rev. B 59, 10959 (1999).

${ }^{21}$ J. Rusz, O. Eriksson, P. Novák, and P. M. Oppeneer, Phys. Rev. B 76, 060408(R) (2007).

${ }^{22}$ J. Rusz, S. Rubino, and P. Schattschneider, Phys. Rev. B 75, 214425 (2007)

${ }^{23}$ J. P. Morniroli, F. Houdellier, C. Roucau, J. Puiggalí, S. Gestí, and A. Redjaïmia, Ultramicroscopy 108, 100 (2008).

${ }^{24}$ The delocalization via the long-range Coulomb interaction is given by the modified Bessel function $K_{0}\left(q_{E} x\right)$, where $q_{E}$ $=k_{0} E / 2 E_{0}$ is the characteristic wave number, i.e., for the $\mathrm{Fe} L$ edge and $200 \mathrm{keV}$ primary electron energy $q_{E}=4.4 / \mathrm{nm}$. The FWHM of the delocalization function is $0.08 \mathrm{~nm}$, and the $10 \%$ level is at $0.6 \mathrm{~nm}$ (Ref. 20).

${ }^{25}$ H. Lidbaum, J. Rusz, A. Liebig, B. Hjörvarsson, P. M. Oppeneer, E. Coronel, O. Eriksson, and K. Leifer, arXiv:0807.1805 (unpublished). 